\title{
NEUROACANTOCTOSE
}

\author{
RELATO DE CASO
}

\author{
ANDRÉ R. TROANO*, PAULOC. TREMSQL-BITIENCOURT **
}

\begin{abstract}
RESUMO - Relatamos o caso de um paciente de 45 anos com neuroacantocitose. O paciente apresenta crises parciais complexas com automatismos e crises generalizadas tônico-clônicas, assim como distúrbios do movimento caracterizados por coréia do tronco e membros superiores, e discinesia orofacial. Os exames complementares revelam acantocitose de $11 \%$, eletrencefalograma com foco irritativo no lobo temporal direito, creatino fosfoquinase sérica de 101 U/L e imagem de ressonância magnética com redução volumétrica e hiper-intensidade do sinal no núcleo caudado e putâmen bilateralmente.
\end{abstract}

PALAVRAS-CHAVE: acantocitose, distúrbios do movimento, epilepsia.

\section{Neuroacanthocytosis: a case report}

ABSTRACT - We present a 45 years old man with neuroacanthocytosis. This gentleman has complex partial seizures and generalized tonic-clonic seizures, as well as movement disorders characterized by chorea and orofacial diskinesia. Complementary examination shows acanthocytosis of $11 \%$ on peripheral blood, irritative focus on right temporal lobe on EEG, serum creatinokinase of $101 \mathrm{U} / \mathrm{l}$ and volume reduction and hypersignal on caudate nucleus and putamen bilaterally on MRI.

KEY WORDS: acanthocytosis, movement disorders, epilepsy.

A neuroacantocitose (NA) é distúrbio neurológico no qual manifestações extrapiramidais, amiotrofia e epilepsia associam-se frequentemente à presença de hemácias espiculadas, denominadas acantócitos, no sangue periférico. Trata-se de enfermidade rara, provavelmente subdiagnosticada, havendo poucos casos relatados na literatura médica. Relatamos o exame clínico, a complementação diagnóstica laboratorial e de imagem, o tratamento e a evolução clínica de paciente com diagnóstico de NA.

\section{REATODOCASO}

JAM, 45 anos, branco, casado, origem açoriana, natural de Biguaçu e procedente de Palhoça, ambos municípios situados na região metropolitana de Florianópolis, frentista aposentado. 1 Este paciente era assintomático até os 6 anos de idade, quando apresentou uma crise generalizada tônico-clônica (CGTC). Aos dezesseis anos repetiu crise com padrão idêntico. Nega uso de qualquer medicação entre estes dois episódios. A partir deste último, o sintoma passou a ser recorrente. Além disso, seus ataques passaram a ter nuances diferentes dois tipos distintos de crises epiléticas sendo identificados. A primeira modalidade, descrita por ele e seus familiares

*Acadêmico da Faculdade de Medicina da Universidade Federal de Santa Catarina (UFSC); **Médico neurologista e professor de Neurologia do Departamento de Clínica Médica do Hospital Universitário (HU), UFSC. Aceite: 30-março-1999.

Dr. Paulo César Trevisol-Bittencourt - Hospital Universitário UFSC - 88040-970 Florianópolis SC - Brasil. E-mail: pcb@hu.ufsc.br 
como ataque, era representada pelas típicas CGTC iniciadas na infância, eventualmente precedidas por sintomas sensitivos mal definidos. A segunda, descrita como ameaça, surgida ao final da adolescência, era uma crise parcial complexa com automatismos (CPC). Estas também eram usualmente precedidas por fenômenos sensitivos. A frequência destas crises era variável, porém, quando da sua primeira avaliação em 1983 na Clínica Multidisciplinar de Epilepsia (CME) tinha média mensal de 4-6 episódios de CPC e 2-4 CGTC.

O senhor JAM é acompanhado na CME desde esta época. Permaneceu relativamente bem por aproximadamente 4 anos, fazendo uso regular de carbamazepina (CBZ) em regime de monoterapia. Após esta longa lua de mel com CBZ, observou-se piora sensível, com aumento na frequência e severidade das crises epiléticas. Devido a isso, CBZ foi suprimida e fenitoína (PHT) foi introduzida no seu lugar. Após 6 meses de relativa calmaria epiléptica, notou-se o surgimento insidioso de uma síndrome extrapiramidal, constituída de movimentos coreicos de tronco e extremidades.

Diante disso, PHT foi incriminada como a responsável e interrompida. Valproato de sódio foi iniciado em substituição. Apesar desta providência, seus movimentos involuntários continuaram sofrendo piora gradativa. Além disso, discinesia orofacial passou a fazer parte do seu quadro. Aliás, parecia que em cada visita ambulatorial, este paciente acrescentava mais um item a sua peculiar síndrome. Sempre um novo sintoma era agregado e os anteriores, piorados. Segundo sua esposa, JAM está ficando muito fraco e agora começou a grunhir e às vezes fica muito brabo e diz cada nome feio... Além disso, ultimamente só anda falando em se matar. Ele foi internado no Hospital Universitário UFSC, para reavaliação da sua condição. Recebeu alta após cerca de 10 dias com o diagnóstico de NA e a recomendação de usar fenobarbital (PB) $100 \mathrm{mg}$ ao dia e haloperidol 10 gotas 3 vezes ao dia. Quando do seu retorno ambulatorial , JAM, além de persistir com sua síndrome extrapiramidal, tinha crises frequentes e uma sensível deterioração cognitiva e comportamental. Decidiu-se então pela reintrodução de CBZ associada a clorpromazina. Na sua última avaliação, há cerca de 3 meses, sua epilepsia estava relativamente bem controlada, todavia seus sinais extra-piramidais continuavam em lenta e gradual progressão. Notamos também que disfagia passou a ser um dos seus piores sintomas e que sua fala transformou-se num jargão de dificílimo entendimento. As palavras mal pronunciadas e a sua baixa tonalidade tornavam-nas incompreensíveis em sua maioria.

Em relação a sua história familial, sua esposa informou que a sogra, já falecida, era portadora de epilepsia (sic) e que um neto, por parte de sua filha, é portador de síndrome de West (sic).

Sua mais recente avaliação clínica evidencia um exame físico geral normal, entretanto, uma semiologia neurológica exuberante é facilmente detectada. JAM apresenta: (1) alentecimento cognitivo moderado, (2) amiotrofia generalizada, (3) movimentos involuntários rítmicos lentos $(1 / 2 \mathrm{~Hz})$ em rotação do tronco, (4) coreoatetose nas mãos, mais exuberante à esquerda, (5) discinesia orofacial, (6) tiques vocais, (7) incoordenação da marcha, (8) arreflexia tendínea e (9) instabilidade postural. O escore do miniexame do estado mental foi 21/30 pontos.

Os principais resultados de provas complementares foram: (1) eletrencefalograma (EEG) apresentando sinais raros de foco irritativo com projeção temporal do hemisfério direito, (2) creatinofosfoquinase sérica (CPK) de 101 U/L, (3) ressonância nuclear magnética (RMN) de crânio exibindo redução volumétrica e hiper-intensidade do sinal no núcleo caudado e putâmen bilateralmente em T2 e (4) hematoscopia com pesquisa de acantócitos revelando $11 \%$ de acantócitos.

\section{DISQUSSÃO}

A neuroacantocitose (coreoacantocitose, coréia amiotrófica, síndrome de Levine-Critchley) é síndrome rara, de início tardio, caracterizada por discinesia buco-línguo-facial levando a automutilação dos lábios e língua, movimentos coreicos de cabeça e membros superiores, crises epiléticas e neuropatia periférica com amiotrofia e arreflexia ${ }^{1}$. Suas primeiras descrições datam da década de 1960 quando Levine, em New England (1960), e Critchley, em Kentucky (1968), descreveram famílias nas quais a acantocitose estava associada a padrão lipoproteico normal e a quadro neurológico típico ${ }^{2,3}$. Entretanto, estes não foram os primeiros relatos de doença neurológica associada a acantocitose. Já em 1950, Bassen e Kornzweig haviam relatado fraqueza dos membros e arreflexia com ataxia sensorial e cerebelar em crianças com abetalipoproteinemia e acantocitose (acantocitose de Bassen-Kornzweig). Esta síndrome pode ser dividida em quatro grupos: (1), NA com lipoproteínas normais (síndrome de Levine-Critchley); (2), NA com hipolipoproteinemia; (3), NA com abetalipoproteinemia (doença de Bassen-Kornzweig); (4), síndrome de McLeod, uma anormalidade na expressão de antígeno do grupo sanguíneo Kell, ligada ao cromossomo $\mathrm{X}^{4}$. 


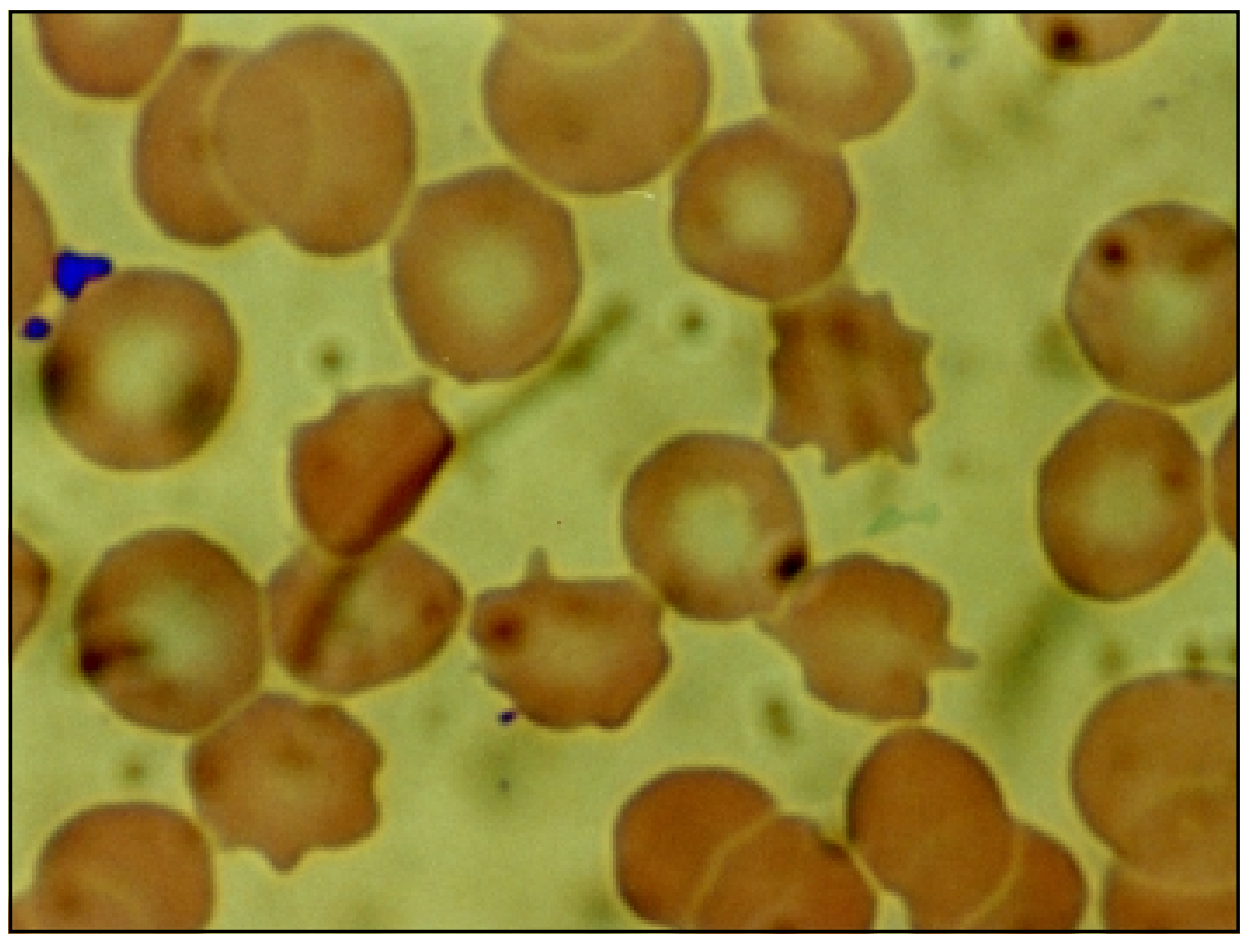

Fig 1. Esfregaço de sangue periférico demonstrando hemácias disformes de aspecto espiculado (acantócitos) (coloração MMG; aumento 1000X).

Em 1981, Sakai et al. sugeriram os seguintes critérios diagnósticos para a identificação desta síndrome: (1) início na vida adulta; (2) discinesia oro-facial progressiva e movimentos coreicos das extremidades; (3) mordedura da língua e lábios, em decorrência da discinesia; (4) neuropatia de desnervação periférica; (5) acantocitose eritrocitária (Fig 1); (6) aumento da creatinofosfoquinase; e (7) herança autossômica recessiva ${ }^{4}$.

Embora a discinesia orofacial e a coréia sejam os distúrbios do movimento clássicos associados com neuroacantocitose, outras modalidades de movimentos anormais involuntários foram relatadas por Hardie et al. ${ }^{5}$ : contrações anormais faringeanas e palatais causando disfagia, disartria e vocalizações involuntárias. Tiques foram observados em 8 dos 19 indivíduos apresentados naquele artigo, distonia em 4, distonia e coréia em 5. Os autores concluem que "as discinesias que ocorrem na neuroacantocitose compreendem a coréia, tiques ou distonia, ou uma combinação destes em vários movimentos anormais".

Apesar de reconhecermos que a síndrome extrapiramidal de nosso paciente era dominada pela coréia, movimentos distônicos e tiques, principalmente vocais, são frequentes. Aliás, nunca soube-se tanto sobre tiques como atualmente, porém, eles ainda permanecem envoltos por uma bruma, podendo estar associados a distintas condições nosológicas. Entretanto, para realçar ainda mais sua importância, destacamos o artigo de Spitz et al. ${ }^{6}$, no qual é notificado o quadro clínico de dois irmãos apresentando tiques faciais e vocais associados a acantocitose do sangue periférico, que foram progressivamente substituídos por parkinsonismo e amiotrofia.

A Tabela 1, extraída de Spitz et al. ${ }^{6}$ e Ferrer et al. ${ }^{7}$, atualizada com as notificações de Miranda et al. ${ }^{7}$, Malandrini et al. ${ }^{1}$ e Serra et al. ${ }^{4}$, contém os principais dados clínicos dos pacientes já descritos.

O exame anátomo-patológico do sistema nervoso central na NA demonstra atrofia esponjosa severa bilateral do núcleo caudado e atrofia cortical leve difusa ${ }^{1}$. JAM evidencia à RMN uma atrofia 
Tabela 1. Principais características clínicas e laboratoriais relatadas na neuroacantocitose.

\begin{tabular}{|c|c|c|c|c|c|c|c|c|c|c|}
\hline Autor & $\begin{array}{l}\text { Idade } \\
\text { de início }\end{array}$ & Sexo & Coréia & Epilepsia & $\begin{array}{c}\text { Problemas } \\
\text { Psíquicos }\end{array}$ & $\begin{array}{c}\text { Arrefle- } \\
\text { xia }\end{array}$ & $\begin{array}{c}\text { Amio- } \\
\text { trofia }\end{array}$ & $\begin{array}{l}\text { Heredita- } \\
\text { riedade }\end{array}$ & & $\begin{array}{c}\% \\
\text { de } \\
\text { acantócitos }\end{array}$ \\
\hline Levine "68 & 27 & M & + & + & + & + & + & $\mathrm{AD}$ & + & $20 \%$ \\
\hline Critchley '67 & 23 & M & + & - & + & + & ? & $\mathrm{AR}$ ? & $?$ & $30-40 \%$ \\
\hline Critchley '70 & 26 & $\mathrm{~F}$ & + & - & + & +- & - & $\mathrm{S}$ & $?$ & $40-60-\%$ \\
\hline \multirow[t]{2}{*}{ Aminoff ' 72} & 58 & M & + & + & - & + & + & AR & + & $25-30 \%$ \\
\hline & 56 & $\mathrm{M}$ & + & - & - & + & - & $\mathrm{AR}$ & - & $25-30 \%$ \\
\hline Bird ' 78 & 40 & M & + & + & +- & + & + & AR & + & $5-15 \%$ \\
\hline Itoga '78 & 36 & M & + & ? & - & +- & + & $\mathrm{S}$ & + & + \\
\hline \multirow[t]{2}{*}{ Kamakura '79 } & 20 & $\mathrm{~F}$ & + & + & - & +- & - & $\mathrm{S}$ & - & + \\
\hline & 20 & M & + & - & - & + & + & $\mathrm{S}$ & + & + \\
\hline Kito ' 80 & 36 & M & + & ? & + & + & + & $\mathrm{AD}$ & + & + \\
\hline \multirow{3}{*}{ Ohnishi ‘ 81} & 29 & $\mathrm{~F}$ & + & + & + & - & - & $\mathrm{AR}$ & + & $30-80 \%$ \\
\hline & 27 & M & + & - & + & + & + & $\mathrm{AR}$ & + & $50-80 \%$ \\
\hline & 29 & M & - & + & + & + & + & $\mathrm{S}$ & + & $12-19 \%$ \\
\hline Sakai ‘81 & 27 & M & + & - & - & + & + & $\mathrm{AR}$ & + & $20-38 \%$ \\
\hline Limos '82 & 28 & M & + & - & + & + & + & $\mathrm{S}$ & + & $41-69 \%$ \\
\hline \multirow[t]{2}{*}{ Yamamoto ' 82} & $2 \quad 34$ & M & + & - & + & + & + & $\mathrm{AR}$ & + & $30-35 \%$ \\
\hline & 34 & $\mathrm{~F}$ & + & + & + & + & - & $\mathrm{AR}$ & + & $30 \%$ \\
\hline \multirow[t]{5}{*}{ Ueno '82 } & 32 & M & + & ? & ? & + & + & $\mathrm{AR}$ ? & + & $45 \%$ \\
\hline & 27 & $\mathrm{~F}$ & + & ? & ? & + & - & AR? & + & $26 \%$ \\
\hline & 28 & M & + & ? & $?$ & + & + & $\mathrm{S}$ & + & $57 \%$ \\
\hline & ? & M & + & ? & ? & + & - & AR? & + & $58 \%$ \\
\hline & 40 & M & + & $?$ & $?$ & + & + & AR? & + & $15 \%$ \\
\hline Sotaniemi '83 & 24 & $\mathrm{~F}$ & + & - & - & + & + & $\mathrm{AR}$ ? & + & $50 \%$ \\
\hline Iwata ' 84 & 20 & $\mathrm{~F}$ & + & + & $?$ & + & + & $?$ & $?$ & $10 \%$ \\
\hline \multirow[t]{2}{*}{ Spitz '85 } & 13 & $\mathrm{M}$ & + & - & - & ? & + & $\mathrm{AR}$ & + & $11 \%$ \\
\hline & 36 & M & + & - & - & + & $?$ & $\mathrm{AR}$ & + & $14,9 \%$ \\
\hline Gross '85 & 36 & $\mathrm{M}$ & + & + & - & + & + & $\mathrm{AR}$ & + & $15-20 \%$ \\
\hline Sobue ' 86 & 40 & $\mathrm{M}$ & + & + & - & + & + & $\mathrm{S}$ & + & $28 \%$ \\
\hline \multirow[t]{2}{*}{ Serra '86 a } & 34 & $\mathrm{~F}$ & + & + & + & + & + & $\mathrm{AR}$ & + & $61 \%$ \\
\hline & 33 & $\mathrm{M}$ & + & - & - & + & + & $\mathrm{AR}$ & + & $30 \%$ \\
\hline \multirow[t]{6}{*}{ Serra ' 86 b } & 50 & $\mathrm{M}$ & + & - & - & + & + & $\mathrm{AR}$ & + & $44 \%$ \\
\hline & 38 & $\mathrm{M}$ & + & + & + & + & + & $\mathrm{AR}$ & + & $19 \%$ \\
\hline & 33 & $\mathrm{M}$ & + & - & + & + & + & $\mathrm{AR}$ & + & $50 \%$ \\
\hline & 34 & $\mathrm{M}$ & + & - & - & + & + & AR & + & $33 \%$ \\
\hline & 29 & $\mathrm{M}$ & + & ? & ? & ? & + & $\mathrm{AR} ?$ & + & $29 \%$ \\
\hline & 47 & $\mathrm{M}$ & + & ? & ? & ? & + & $\mathrm{AR}$ ? & + & $23 \%$ \\
\hline \multirow[t]{4}{*}{ Vance '87 } & 32 & $\mathrm{M}$ & + & - & +- & + & - & $\mathrm{AR}$ & + & $10 \%$ \\
\hline & 43 & $\mathrm{~F}$ & + & - & + & + & + & AR & + & $2 \%$ \\
\hline & 35 & $\mathrm{M}$ & + & - & + & + & - & $\mathrm{S}$ & + & $30 \%$ \\
\hline & 23 & $\mathrm{~F}$ & + & + & + & + & - & $\mathrm{S}$ & + & $10 \%$ \\
\hline \multirow[t]{2}{*}{ De Yebenes ' 88} & $38 \quad 27$ & M & + & + & + & ? & + & $\mathrm{AR}$ & $?$ & $20-36 \%$ \\
\hline & 18 & $\mathrm{M}$ & + & + & + & ? & ? & $\mathrm{AR}$ & $?$ & $30-40 \%$ \\
\hline \multirow[t]{2}{*}{ Dubinsky ‘89 } & 33 & $\mathrm{M}$ & + & + & + & ? & ? & $\mathrm{AR}$ ? & $?$ & $20-30 \%$ \\
\hline & 30 & $\mathrm{M}$ & + & + & $?$ & ? & $?$ & $\mathrm{AR}$ ? & $?$ & $20-30 \%$ \\
\hline Bharucha ' 89 & 42 & $\mathrm{~F}$ & + & - & - & + & - & $?$ & - & $?$ \\
\hline Medalia '89 & 27 & $\mathrm{~F}$ & + & + & + & + & - & ? & + & ? \\
\hline Ferrer '90 & 32 & M & + & + & + & + & - & $\mathrm{AR}$ ? & + & $10-20 \%$ \\
\hline \multirow[t]{3}{*}{ Miranda '93 } & 22 & $\mathrm{~F}$ & + & - & - & + & - & $\mathrm{S}$ & + & $25 \%$ \\
\hline & 45 & M & + & - & - & + & + & $\mathrm{S}$ & + & $20 \%$ \\
\hline & 25 & M & + & - & + & - & + & AR? & + & $15-30 \%$ \\
\hline \multirow{2}{*}{ Malandrini '93 } & $\begin{array}{ll}3 & 17\end{array}$ & M & + & + & + & + & + & $\mathrm{S}$ & + & 0 \\
\hline & 23 & M & + & + & + & + & + & $\mathrm{AR}$ & + & $11 \%$ \\
\hline $\begin{array}{l}\text { Nossa } \\
\text { observação }\end{array}$ & 40 & $\mathrm{M}$ & + & + & + & + & + & S? & + & $11 \%$ \\
\hline
\end{tabular}

Adaptado de Ferrer et al. ${ }^{10}(1990)$. 
importante de caudado e putâmen bilaterais, com córtex normal. Atribui-se essa atrofia nuclear ao resultado da rarefação neuronal associada à gliose astrocitária. Estes achados são compatíveis com resultados de exames de neuroimagem, nos quais a atrofia do núcleo caudado, habitualmente poupando os demais núcleos da base, associa-se a atrofia cortical leve. Miranda et al. ${ }^{8}$ sugerem que a existência de um defeito em determinadas proteínas de membrana levaria a alteração de permeabilidade, ocasionando disfunção e morte celular. Esta alteração poderia ser especialmente deletéria nos eritrócitos e nos neurônios estriatais. Teoria semelhante era proposta por Levine et al. ${ }^{2}$ já em 1968, ao afirmarem que "é muito provável que tanto as manifestações do sistema nervoso quanto as alterações nas hemácias sejam expressões de anormalidades em sistemas enzimáticos básicos transmitidos como herança autossômica dominante" (a família relatada pelo autor em 1968 apresentava distúrbios do movimento associados à acantocitose transmitidos como padrão autossômico dominante. Em sua maioria, os relatos subsequentes apresentaram padrão autossômico recessivo).

Em estudo bioquímico detalhado sobre as modalidades de defeitos em componentes da membrana eritrocitária e definição de testes de estresse para hemácias, Feinberg et al ${ }^{9}$ postulam que compostos que se inserem na camada externa da membrana das hemácias, como os salicilatos e os ácidos graxos, causam transformação equinocítica, levantando à "hipótese do acoplamento da bicamada". Esta teoria afirma que a expansão desigual dos folhetos externos da membrana da hemácia leva à equinocitose, ou seja, às hemácias espiculadas observadas à hematoscopia. Há, neste artigo, sugestão sutil de que a classificação hematológica adequada para a anormalidade observada nas hemácias deste pacientes é a equinocitose. Esta diferenciação baseia-se no fato de que os acantócitos, observados na doença hepática e na abetalipoproteinemia de Bassen-Kornzweig, não se transformam novamente em discócitos, transformação esta que é notada nas hemácias da neuroacantocitose.

Sabe-se que a clorpromazina age sobre o folheto interno da bicamada lipídica e provavelmente expande a superfície interna do eritrócito. Concentrações plasmáticas crescentes de clorpromazina resultam em invaginação progressiva da membrana, de forma a reverter a transformação equinocítica. Em baixas concentrações de clorpromazina as células parecem discócitos normais e, em concentrações maiores, progridem a estomatócitos ${ }^{3}$. O senhor JAM fez uso deste fármaco por prolongado período de tempo, mas não podemos afirmar definitivamente se a clorpromazina provocou menor contagem de acantócitos do que a que se observaria sem este neuroléptico.

Em parte da literatura disponível sobre este assunto, é proposta a necessidade de um mínimo de $15 \%$ de acantocitose no sangue periférico como critério diagnóstico da coreoacantocitose. Sustentados nos dados da literatura atual revisada, propomos a reavaliação deste conceito. Malandrini et al. ${ }^{1}$ e Feinberg et al. ${ }^{9}$ relatam casos de pacientes nos quais o quadro clínico indistinguível da apresentação clássica da NA e do paciente que ora apresentamos não estavam associados a acantocitose. Além disso, Feinberg propõe testes de estresse mecânico e osmótico para facilitação da observação de acantocitose. São estes: (1) observação sob lamínula em diluição salina, (2) diluição em salina normal ou tamponada com fosfato e fixada e (3) envelhecimento da amostra de sangue em EDTA por 0-7 horas. Todos estes testes promovem a transformação de discócitos em equinócitos no sangue de indivíduos normais. No entanto, em pacientes com NA ou quadro clínico compatível em investigação sem acantocitose evidente, a anormalidade é um aumento dramático da sensibilidade das hemácias ao estresse equinocítico.

Um outro aspecto relevante no paciente reportado é a historia familial positiva para epilepsia, com mãe e neto acometidos. No entanto, estes não puderam ser examinados pessoalmente, de forma que evitamos especulação quanto à possibilidade de existência de herança genética. Embora a epilepsia faça parte do quadro da coréia-acantocitose em muitos indivíduos, Meierkord e Shorvon foram os primeiros autores a dedicar exclusivamente a ela um artigo ${ }^{10}$. Segundo estes, as crises não raramente precedem o estado hipercinético em meses a anos. Mesmo depois do desenvolvimento de síndrome extrapiramidal, a epilepsia pode constituir o principal problema e o maior desafio terapêutico nestes pacientes. De maneira análoga ao apresentado por JAM, estes autores observaram predominantemente ataques tônico-clônicos primariamente generalizados e parciais complexos com generalizações secundárias? 
Finalmente alguns comentários sobre a polineuropatia evidenciada clinicamente pelo nosso paciente. Achados à biópsia do nervo sural sugerem que a polineuropatia da NA é devida a axonopatia distal. Notou-se perda severa de fibras mielinizadas, sendo poupados os neurônios motores espinhais e as células ganglionares. $\mathrm{O}$ acúmulo de neurofilamentos no axoplasma dos nervos surais e nos axônios da coluna dorsal da medula espinhal sugerem que o transporte axonal diminuído pode ser responsável por este tipo de neuropatia ${ }^{1}$. No momento, a polineuropatia periférica é aparentemente o maior adversário de JAM, produzindo limitação motora progressiva.

Gostaríamos de enfatizar que pacientes com NA parecem ter um potencial suicida similar ao observado originalmente nos sofredores de doença de Huntington. Nosso paciente frequentemente faz alusão a esta alternativa. Assim, sugerimos que seja dada atenção especial a este aspecto quando o diagnóstico de NA estiver sob suspeição ou for estabelecido.

A NA é uma doença rara do sistema nervoso central e periférico, em que movimentos anormais, amiotrofia e arreflexia associam-se quase sempre a alterações morfológicas nas hemácias dos pacientes afetados. Alterações neuropáticas são frequentes. A epilepsia é uma comorbidade frequente. Mais comumente, é uma doença familial, com herança autossômica recessiva. Deve-se ter esta hipótese diagnóstica em mente frente a paciente com movimentos involuntários anormais de início na vida adulta, associado a epilepsia, amiotrofia e arreflexia generalizadas. A história familiar é frequentemente positiva. Nestes casos, a pesquisa por acantócitos no sangue periférico por hematologista experiente é recomendada. Esta condição neurodegenerativa pode ainda ser atualmente subdiagnosticada, e sua presença no hall das enfermidades que causam início de movimentos involuntários anormais na adolescência tardia e vida adulta pode proporcionar maior influxo de relatos de casos e descrições de famílias no hemisfério sul e especialmente na América do Sul. Após as publicações originais, este quadro foi comunicado principalmente no Japão, Europa e América do Norte, com apenas duas notificações de pacientes latino-americanos. Este é o terceiro relato de coreoacantocitose na América Latina, e o primeiro no Brasil.

Agradecimentos - A realização deste artigo não teria sido possível sem a ajuda do Dr. Antônio Carlos Scaramello (Disciplina e Serviço de Anatomia Patológica, HU, UFSC), Dr. Pedro Lemos de Luca (Clínica Radiológica Dr. Carlos Corrêa) e Dr. Gilmar Pacheco (Disciplina de Hematologia, Departamento de Clínica Médica, HU, UFSC).

\section{REFERÊNAAS}

1. Malandrini A, Fabrizi GM, Palmeri S, Ciacci G, Salvadori C, Berti G. Choreo acanthocytosis like phenotype without acanthocytosis: clinicopatological case report. Acta Neuropathol 1993;86:651-658.

2. Levine IM, Estes JV, Looney JM. Hereditary neurological disease with acanthocytosis: a new syndrome. Arch Neurol 1968;19:403-409.

3. Critchley EMR, Clark DB, Winkler A. Acanthocytosis and neurological disorder without abetalipoproteinemia. Arch Neurol 1968;18:134-140.

4. Serra S, Xerra A, Arena A . Amyotrophic chorea-acanthocytosis: a new observation in southern Europe. Acta Neurol Scand 1986;73:481-486.

5. Hardie RJ, Pullon HWH, Harding AE, Owen JS, Pires M. Neuroacanthocytosis: a clinical, haematological and pathological study of 19 cases. Brain 1991;114:13-49.

6. Spitz MC, Jankovic J, Killian JM. Familial tic disorder, parkinsonism, motor neuron disease and acanthocytosis: a new syndrome. Neurology 1985;35:366-370.

7. Ferrer X, Julien J, Vital C, Lagueny A, Tison F. La chorée-acanthocytose. Rev Neurol 1990;146:739-745.

8. Miranda M, Campero M, Tenhamm E, Villagra R. Neuroacantocitosis: comunicacion de 3 casos. Rev Méd Chile 1993;21:176-179.

9. Feinberg TE, Cianci CD, Morrow JS, et al.. Diagnostic tests for choreoacanthocytosis. Neurology 1991;41:1000-1006.

10. Meierkord H, Shorvon S. Epilepsie bei Neuroakanthozytose. Nervenarzt 1990;61:692-694.

11. Aminoff MJ. Acanthocytosis and neurological disease. Brain 1972;95:749-760.

12. Gross KB, Strivanek JA, Carlson KC, Kaufman DM. Familial amyotrofic chorea with acanthocytosis: new clinical and laboratory investigations. Arch Neurol 1985;42:753-756.

13. Kito S, Itoga E, Hiroghige Y, Matsumoto N, Miwa S. A pedigree of amyotrophic chorea with acanthocytosis. Arch Neurol 1980;37:514-517.

14. Mc Lellan DL, Swash M, Choreo-athetosis and encephalopathy induced by phenitoin. Br Med J 1974;2:204-205. 